\title{
NEURAL BASIS OF THE SPONTANEOUS OPTOKINETIC RESPONSE PRODUCED BY VISUAL INVERSION
}

\author{
R. W. SPERRY \\ Hull Laboratory of Anatomy, The University of Chicago ${ }^{1}$ \\ Received for immediate publication August 6, 1950
}

One of the most conspicuous behavioral effects produced by surgical rotation of the eyeball through 180 degrees is the forced circling or spontaneous optokinetic reaction. Animals with inverted vision caused by eye rotation tend to turn continuously in circles $(4,6,8)$. In fishes and amphibians this circling may persist indefinitely with little or no correction by reeducation. Similar circus movement is caused by contralateral transplantation of the eyeball with inversion on only one axis and also by cross-connection of the optic nerves to the wrong side of the brain (7). In all these situations movement on the part of the animal causes the visual image to pass over the retinal field or its central projection in a direction opposite to normal.

The forced circling phenomenon of inverted vision is an illustration of the importance of kinesthetic effects in visual perception. Under normal conditions the image of the visual field, whether it be stationary or moving across the retina, can be perceived as being either in motion or at rest depending upon whether the perceiving animal is moving or at rest. The apparent position and direction of motion of the visual field depend upon the posture and movements of the animal as well as upon the patterning of the retinal stimulus itself. It has been suggested that eye rotation disturbs the normal interrelation of the retinal and the non-optic, kinesthetic components of visual adjustment so that the latter, instead of acting in the normal manner to maintain constancy of the visual field when the animal is moving, tend rather to accentuate the illusion of outside motion. Once started, the illusory drifting of the visual field presumably sets off the typical optokinetic response which, in cyclic fashion, further enhances the illusory movement. The result is a spontaneous circling reaction quite similar to the optokinetic response produced by actual rotation of the visual field.

The foregoing interpretation is supported by the subjective reports of persons who have worn inverting oculars. Body and head movements are said to cause the entire visual scene to sweep and swim before the eyes with resultant giddiness and nausea. The inverted surroundings appear to drift in the same direction as, but more rapidly than, the subjects's own movement $(10,11)$.

The importance of the central mechanisms underlying these phenomena for the normal visual perception of space and motion prompted an attempt to find out something about the neural apparatus involved, using the forced circling of eye rotation as an indicator. The investigation was started with teleost fishes in which the circling phenomenon is particularly pronounced. In addition to various advantages which the fishes offer with respect to surgery, maintenance, and observation, the brain is comparatively simple and is much less susceptible to diaschisis than is that of the higher vertebrates.

\section{MATERIALS AND METHODS}

Eye rotation was combined with ablation of various brain centers and extirpation of the otic labyrinths in an attempt to determine the respective roles, if any, played by each

1 This investigation was carried out at the Lerner Marine Laboratory, Bimini, B. W. I., and the Bermuda Biological Station, Bermuda. 
in the forced circling reaction. Sphaeroides spengleri (Bloch), the southern swellfish, measuring 7 to $9 \mathrm{~cm}$. in total length, was used throughout. Its small size, loose, scaleless integument, and general hardiness make this fish suitable for experiments involving surgery. The marked development of its visual system, the easy accessibility of its brain, its habit of resting frequently on the bottom, and of swimming with comparatively little body movement, added to the above, make this species particularly favorable for the present experiments. The fish were collected during February and March and were kept in groups of three to five in aquaria, approximately 55 by 35 by $30 \mathrm{~cm}$., with running sea water.

Following their anesthetization in a 1.5 per cent solution of urethane, the fish were operated upon out of water under a dissecting microscope with a magnification of 20 times. Rotation of the eyeball was performed through a dorsal incision into the orbit. All extraocular muscles were severed and all other connections of the eyeball were broken except the optic nerve and main blood vessels. The eyeball was then rotated anterodorsally and oriented at 180 degrees from the normal resting position. A similar approach was used for cutting the extraocular muscles of the contralateral eye, and for inserting blinders of tantalum foil.

Removal of brain parta was performed by aspiration. The forebrain, optic tectum, and cerebellum were extirpated through openings in the roof of the cranium directly over the part to be removed. The inferior lobes of the infundibulum were approached ventrally via the expansible diverticulum of the stomach, the esophagous, the pharynx, and a median opening in the floor of the cranium. The bone was not replaced and sheets of connective tissue gradually formed over the openings in the cranium. All skin incisions were closed with sutures of fine silk. Losses from infection were negligible although only minor precautions were taken to maintain asepsis during operation. At the termination of the experiments the brain lesions were checked by dissection under a microscope following formalin fixation. No cases are included below in which the lesions were found to have been less extensive than intended.

An interval of at least 2 days was allowed between serial operations performed on the same animal. Two or more days were allowed also for the temporary effects of the surgery and anesthesia to disappear before drawing conclusions regarding the functional effects of an operation. Unless otherwise stated in context, these conclusions are based in each instance on a minimum of 4 cases.

\section{OPERATIONS AND RESULTS}

The left eye was rotated 180 degrees, as described above, in a series of 37 fish. The right eye was blinded in 8 of these cases by section of the optic nerve, in 5 others by implantation of tantalum eye caps (9), and, in the remainder, by stitching the circumorbital skin over the eye. (The skin around the eyeball is extremely loose in these fish and is naturally drawn over the eye in purse-string fashion in the corneal reflex.)

The forced circling produced by unilateral visual inversion was not consistently one-sided as in the amphibians. These fish circled readily either to right or left. Apparently each individual tended to circle in the direction in which it happened to get started. Occasionally a fish would shift the direction of its circling from one day to the next. The circling occurred predominantly in the frontal plane although somersaulting about the right-left body axis, rolling on the longitudinal axis, and intermediate combinations also were displayed, particularly during excitement. The diameter of the circles in this species was usually slightly less than the total length of the fish and it varied but little over the entire range of speeds at which the fish swam.

Unpublished observations had shown that removal of the forebrain has no 
appreciable effect on the simple aquarium behavior of this species. Accordingly, forebrain ablation would not be expected to cause any disturbance of the forced circling of inverted vision. The results were as anticipated; complete bilateral removal of the forebrain including the olfactory tracts left the circling behavior unaffected.

The well-known correlation of cerebellar lesions with disequilibrium and nystagmus in the higher vertebrates, along with the recent demonstrations of visual representation in the cerebellar cortex (3) indicated the cerebellum as a possible center for integration of retinal and kinesthetic patterns. However, the effects of removal of the cerebellum proved to be much the same as removal of the forebrain with no specific impairment of the forced circling reaction. Even with both cerebellum and forebrain excised, whether serially or simultaneously, the fish continued to circle in typical manner. Corollary tests showed that combined ablation of forebrain plus cerebellum in fish otherwise normal produced no abnormality in their aquarium behavior that was evident to casual observation 3 weeks after operation.

Excision of the extraocular muscles on the blind side in the forebrainless, cerebellumless fish with rotated eyes did not disturb the circus movements. The rotated eye was returned to its normal position in these cases in a control procedure. This caused cessation of the forced circling.

The inferior lobes of the hypothalamus are extremely large in $S$. spengleri. The fiber tract associations suggest that these lobes are concerned mainly with olfactory and gustatory functions (2). In the absence of more thorough knowledge of their functions, however, it could not be concluded a priori that they were not involved in the spontaneous optokinetic reaction. Accordingly bilateral ablation of the inferior lobes was performed from the ventral approach described above. Removal of the inferior lobes alone did not disrupt the circling response. In 3 cases in which the forebrain plus cerebellum had been removed previously, the additional excision of the inferior lobes also failed to abolish the circling. The swimming of these latter cases was definitely less vigorous than normal, but there appeared to be no essential breakdown of the circling mechanism itself. The circling ceased, however, when the normal orientation of the rotated eye was restored in a final control check.

In all of the foregoing cases in which more than the forebrain had been excised, blinding of the unrotated eye had been effected without sectioning the optic nerve. The asymmetry in brain activity caused by unilateral optic nerve section was not appreciable so long as most of the remainder of the brain and the vestibular systems remained intact. However, trial tests indicated that this asymmetry could become a confusing factor in the presence of drastic lesions in other parts of the brain, or after removal of the labyrinths.

The only major lobes of the brain which had not been removed in the above animals were the optic lobes (see figure 1). In fishes with the left eye rotated, right eye blinded, and the brain otherwise intact, excision of both optic lobes ( 2 cases) or of the right optic lobe alone (3 cases) was effective in abolishing the circling response. 
In 3 fish with the left eye rotated and the right eye blinded by insertion of a tantalum foil cap, removal of the blinder was followed by cessation of the characteristic circling behavior. Apparently the fish responded to the stimuli entering the up-right eye while inhibiting the effects of those entering the rotated eye. Excision of the left optic lobe in all 3 of these cases resulted in a resumption of the circling reaction which continued until the rotated eye was returned to its normal orientation 2 days later.

It was assumed a priori that the extra-retinal kinetic factor involved in the circling behavior had its origin in kinesthetic receptors in the periphery. The intimate association of the vestibular system and vision, as evidenced in the swimming of the visual field during rotation dizziness, and also the paramount role which is played by the labyrinths in maintaining equilibrium in the lower

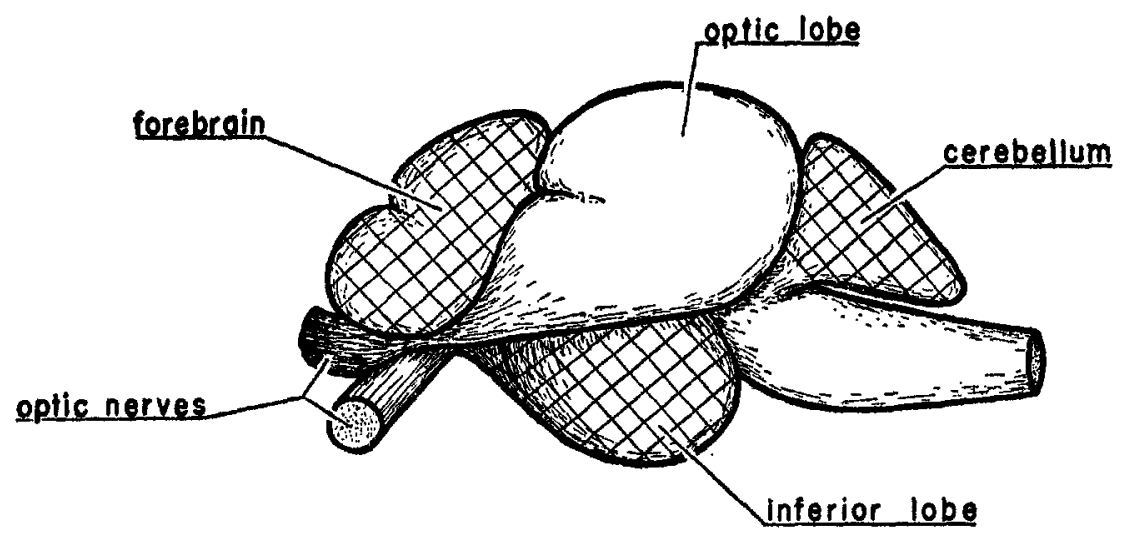

FIa. 1. Diagram of the Brain of $S$. spengleri. Crosshatched areas indicate the parts removed without disrupting the spontaneous optokinetic reaction.

vertebrates, suggested the vestibular system as the most likely source of the kinesthetic component.

The labyrinth was removed bilaterally in a series of 8 fish otherwise normal. In contrast to the effects of ablating forebrain and cerebellum, bilateral labyrinthectomy caused severe motor discoordination and disturbance of equilibrium. During the first 24 hours after operation the fish remained at rest on the bottom. When forced to move, they were unable to swim upright or straight. They somersaulted, spiraled, rolled, and jerked in spasmodic gyrations. Occasionally these gyrations included circling which resembled that of inverted vision, but it was not so regular or consistent. During the next few days the labyrinthectomized fish regained the ability to swim upright so long as they swam very slowly and did not use body and tail movements. Any attempt to move quickly resulted in loss of control. Even their slow swimming was very unsteady and considerable effort seemed necessary to maintain the upright position. In 3 cases maintained as long as 24 days after operation only slight improvement was evident at the end of this period. 
The left eye in 4 of the above 8 cases was rotated and the right eye was blinded 5 days after labyrinthectomy. Even slight asymmetry in the sensori-neuromotor system of the labyrinthectomized fish had been found in trial tests to cause circling and spinning that could be easily confused with the effects of inverted vision. Accordingly an effort was made to keep the trauma on each side equal, as far as possible. When the right eye was blinded, the extraocular muscles were severed as on the left side and the circumorbital skin was stitched together over the eye. Under these conditions the eye rotation resulted in circling quite characteristic of inverted vision. In 3 of the 4 cases, returning the eyeball to its normal position abolished the circling. The circling did not cease immediately as soon as the fish began to recover from anaesthesia as in previous cases, but it had disappeared by the end of the following day. In the 4th case the circling may have been due originally to accidental damage to the optic nerve during eye rotation. Bilateral blinding following complete labyrinthectomy in control cases caused an increase in disequilibrium accompanied by circling and spinning but the circling was not so consistent as that produced by inverted vision.

\section{DISCUSSION}

The forebrain, cerebellum, and inferior lobes may all be eliminated as the primary center for integration of retinal and kinetic components, since the combined ablation of all 3 failed to disrupt the forced circling. Of the brain centers remaining, the optic tectum seems by far the most likely site of the integrative center. It is large and highly differentiated in these forms, and is the main terminal of the optic nerve. Optic fibers terminate also to a lesser degree among the thalamic nuclei. However, the abolishment of the circling by ablation of the optic lobe contralateral to the rotated eye and, conversely, elicitation of the reaction by ablation of the optic lobe of the unblinded contralateral eye both point to the optic tectum as the controlling center. The results do not exclude the possibility that the optic lobe serves merely as an afferent waystation through which the retinal impulses must pass on their way to an integrating center at some lower level of the neuraxis. Although not ruled out by the evidence, this seems unlikely in view of the sensory character of the phenomenon.

Survival of the optokinetic circling after bilateral labyrinthectomy was unexpected. Combined with the brain lesion data it might be taken to indicate that the circling phenomenon was purely optic and not dependent upon any extraretinal kinetic factor. An interpretation along these lines could be conceived as follows: With the eye rotated, any movement that is visually initiated or guided, like that involved in centering the gaze on a peripheral object, would only exaggerate, instead of resolving, the stimulating situation which induced it. This in turn would reinforce in cyclic fashion more of the same (erroneous) response. This interpretation has an advantage in that it can be expressed in purely reflex terms without requiring the assumption of an illusory spinning sensation in the animal.

It is difficult, however, to believe that the smooth, continuous forced circling 
which is made consistently in the same direction and which simulates exactly the circling produced by actual rotation of the visual field could be merely a repeatedly frustrated attempt to orient with reference to some visual object or a succession of such objects. Such an interpretation meets with further difficulties when applied to the urodele amphibians like Triturus. These animals make localizing and orientating movements of the head in any direction across the visual field. The spinning reactions of inverted vision, on the other hand, occur only toward one side, the direction of which correlates consistently with that of the circling produced by actual rotation of the visual scene (4-6). It is not unreasonable to assume, therefore, that an illusory spinning of the visual field, such
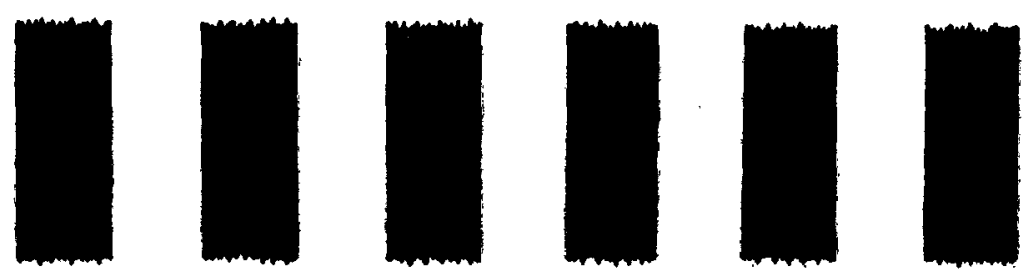

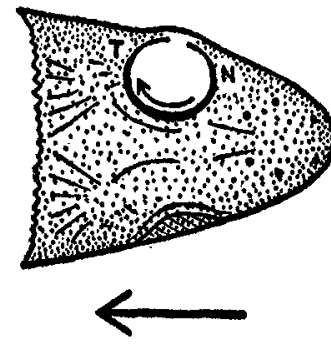

EYE NORMAL

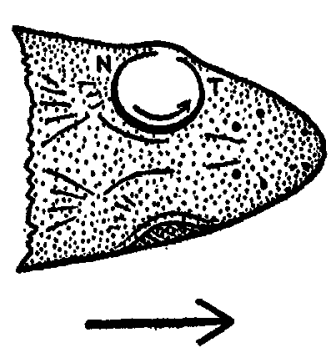

EYE INVERTEO

Frc. 2. Normal Fish Swimming Backward is Subjected to Same Retinal Stimulus as Fish with Rotated Eye Moving Forward. The same retinal influx that does not cause spinning in the former case, does in the latter because of the direction of movement with which it is combined. $N$ and $T$ mark nasal and temporal poles of the eye.

as accompanies visual inversion in man $(10,11)$, is present also in the lower vertebrates and that this is primarily responsible for the forced circling.

Assuming the whirling sensation to be present, it clearly could not result from the retinal stimulation in and of itself. Retinal inversion can exist only with reference to extra-retinal surroundings. It is the inversion with reference to the body and its movement (as registered in the nerve centers) that is crucial. Exactly the same pattern of excitations entering the brain from the retina may, in one instance, arouse the spinning reaction and in another, not, depending entirely upon the direction of the animal's movement accompanying the retinal influx. This is illustrated in Figure 2. It applies to the whirling sensation in man as well as to the overt spinning of the fish. Thus, the direction of the movement 
of the perceiving animal is one of the critical factors that condition the central effects of the retinal stimulus.

It is generally assumed that direction of movement and other kinetic effects are registered centrally via afferent impulses from various kinesthetic receptors in the periphery. In the present experiments it is conceivable that kinesthetic impulses from other receptor sources may have been sufficient in the absence of the labyrinths to maintain the circling mechanism. However, if kinesthetic influx from the periphery were of primary importance, it is surprising that labyrinthectomy did not cause greater disturbance, especially in view of the dominant role played by the labyrinths in these animals.

Another possibility must be considered, namely, that the kinetic component may arise centrally as part of the excitation pattern of the overt movement. Thus, any excitation pattern that normally results in a movement that will cause a displacement of the visual image on the retina may have a corollary discharge into the visual centers to compensate for the retinal displacement. This implies an anticipatory adjustment in the visual centers specific for each movement with regard to its direction and speed. A central adjustor factor of this kind would aid in maintaining stability of the visual field under normal conditions during the onset of sudden eye, head, and body movement. With the retinal field rotated 180 degrees, any such anticipatory adjustment would be in diametric disharmony with the retinal input, and would therefore cause accentuation rather than cancellation of the illusory outside movement.

The postulation of such a central kinetic factor would help account also for certain other phenomena in movement perception such as the illusory displacement of the visual field in man when the eyeball is moved passively as when tapped with the finger tips and the lack of such displacement when the same movement of the eye in space is brought about through an active response. It would provide a neural basis for what Helmholtz (1) called the sensation of the "intensity of the effort of will." The need for some kind of central mechanism to eliminate blurring of vision between fixations in eye movement has long been recognized. The suggestion made with reference to man, that the blur is present but is suppressed by learning is somewhat less applicable to the fishes, many of which including $S$. spengleri display very elaborate eye movements of the exploratory type, even after forebrain and cerebellar ablation. Also, any mechanism that merely suppresses perception in a non-directional fashion during eye movement would not be adequate for maintaining the constancy of the visual field under normal conditions nor the spinning produced by visual inversion.

For the accurate perception of motion and spatial relations it is necessary that the visual centers be kept in constant adjustment with reference to the specific posture of the body and to the speed and direction of all its movements insofar as these affect the visual field. We may imagine the background excitatory state of the visual brain field undergoing continuous fluctuation of an over-all gradient type in response to any movement or shift of posture that affects the direction of gaze. Similar adjustment of the sensorium may be in. 
volved as well in the perception of movement and spatial relations in other sense modalities such as audition and cutaneous sensibility. The indication in the present findings that the postural and kinetic adjustment may have a central origin in part need imply no great decrease in the general importance of afferent excitation from kinesthetic and postural receptors in the periphery. These must continue to be regarded as furnishing an important basic background set or frame of reference for all space perception whether it be principally visual, auditory, or cutaneous.

\section{SUMMARY}

1. The forced circling produced by visual inversion was found to survive bilateral ablation of the forebrain, the cerebellum, or the inferior lobes of the infundibulum; and also the combined ablation of any 2 or of all 3 . It also survived bilateral extirpation of the labyrinths plus severance of the extraocular muscles. It was abolished in the foregoing cases when the eye, as a control check, was returned to its normal orientation.

2. Ablation of the optic lobe of the rotated eye abolished the circling phenomenon. Conversely, the circling could be evoked in animals that had only one eye rotated, by ablating the optic lobe of the contralateral eye.

3. The findings point to the optic lobe as the primary integrative center. They also raise the possibility that a corollary discharge of motor patterns into the sensorium may play an important adjustor role in the visual perception of movement along with non-retinal kinesthetic and postural influences from the periphery.

\section{REFERENCES}

1. Helmholtz, H. von: Physiological optics. Ed. J. Southall. Menasha, Wis.: Banta Pub. Co., 1925. Vol. III.

2. Herrick, C. J.: The central gustatory paths in the brains of bony fishes. $J$. comp. Psychol., 1906, 15, 375-456.

3. SNIDER, R. S. AND STOWELl, A.: Receiving areas of the tactile, auditory, and visual systems in the cerebellum. J. Neurophysiol., 1944, 7, 331-357.

4. SperRY, R. W.: Effect of 180 degree rotation of the retinal field on visuomotor coordination. J. exp. Zool., 1943, 92, 263-279.

5. - : Visuomotor coordination in the newt (Triturus viridescens) after regeneration of the optic nerve. $J$. comp. Neurol., 1943, 79, 33-55.

6. - - Optic nerve regeneration with return of vision in anurans. $J$. Neurophysiol., $1944,7,57-69$.

7. - Restoration of vision after crossing of optic nerves and after contralateral transplantation of eye. $J$. Neurophysiol., 1945, 8, 15-28.

8. - - Patterning of central synapses in regeneration of the optic nerve in teleosts. Physial. Zool., 1948, 21, 351-361.

9. - AND CLARK, E.: Interocular trangfer of visual discrimination habits in a teleost fish. Physiol. Zool., 1949, 22, 372-378.

10. Stratton, G. M.: Some preliminary experiments on vision without inversion of the retinal image. Psychol. Rev., 1896, 3, 611-617.

11. - Vision without inversion of the retinal image, Psychol. Rev., 1897, 4, 341-360, $463-481$. 